\title{
Interferências do fator cor da pele na percepção térmica de transeuntes
}

\author{
Interferences of skin colour on pedestrians' thermal \\ perception
}

\section{Eduardo Leite Krüger \\ Patricia Regina Chaves Drach}

\section{Resumo}

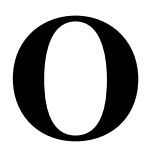

estudo analisa os efeitos da cor da pele na percepção térmica de transeuntes em espaços abertos. O estudo de campo foi realizado no centro da cidade do Rio de Janeiro, tendo sido analisados votos de sensação e preferência térmica de transeuntes. Respostas obtidas por meio de entrevistas estruturadas foram comparadas a resultados do índice de conforto em espaços abertos UTCI (Universal Thermal Climate Index). Os entrevistados foram agrupados de acordo com a autoclassificação quanto à cor da pele segundo o IBGE (Categorias Raciais nos Censos Demográficos). As campanhas de monitoramento foram realizadas em períodos de primavera/verão ao longo de 2012 a 2015, entre 10h e 15h. Utilizou-se uma estação meteorológica Davis Vantage Pro2 para a coleta de dados concomitantemente à aplicação de questionários estruturados segundo a ISO 10551, nos quais a questão quanto à cor da pele do entrevistado foi incluída. Comparando-se sensação e preferência térmicas sob o aspecto de cor da pele, observa-se que, embora o grupo de pessoas de cor da pele branca tenha demonstrado maior tolerância ao estresse térmico por calor, ele mostra preferência mais acentuada por condições térmicas mais frias, de modo a neutralizar tal desconforto. Observaram-se diferenças estatísticas apenas na preferência térmica entre os grupos analisados.

Palavras-chaves: Clima urbano. Percepção térmica em espaços abertos. UTCI. Cor da pele.

Eduardo Leite Krüger Universidade Tecnológica Federal do Paraná

Curitiba - PR - Brasil

Patricia Regina Chaves Drach Universidade do Estado do Rio de

Janeiro Rio de Janeiro - RJ - Brasil

Recebido em 25/03/16 Aceito em 28/06/16

\begin{abstract}
This study analyses effects of skin colour on pedestrians' thermal perception in outdoor spaces. The field study was carried out in downtown Rio de Janeiro, evaluating thermal sensation and thermal preference votes of passers-by. Responses from structured questionnaires were compared to data from the outdoor comfort index UTCI (Universal Thermal Climate Index), by means of data grouping according to skin colour categories provided by the Brazilian Statistical Institute IBGE. In this case, skin colour was informed by the interviewees. The monitoring took place during late spring/summer periods between 2012 and 2015 , typically from 10am until 3pm. A Davis Vantage Pro2 weather station was used while, at the same time, the researchers administered a structured questionnaire based on the ISO 10551, to which a question regarding skin colour was added. The comparison between thermal sensation and thermal preference from the point of view of skin colour demonstrated that while the group with white skin showed greater tolerance to heat, they preferred cooler conditions to help neutralise heat stress. Statistical differences were only observed in the aspect of thermal preference between the groups analysed.

Keywords: Urban climate. Thermal perception in outdoor spaces. UTCI. Skin colour.
\end{abstract}




\section{Introdução}

A aclimatação pode ser definida como mudanças fisiológicas ou comportamentais que ocorrem durante o tempo de vida de um organismo, de modo a reduzir o estresse fisiológico ocasionado por alterações no estresse térmico ${ }^{1}$ no clima natural (TOCHIHARA et al., 2012). Do ponto de vista fisiológico, a regulação térmica do corpo ocorre em diversos campos, incluindo a percepção e a transmissão da informação de ganho ou perda de calor do corpo ou para o corpo, controle dos fluxos de calor entre o corpo e a superfície da pele por circulação cutânea, e geração espontânea de suor para aumento do resfriamento evaporativo, entre outros (JABLONSKI, 2004).

Do ponto de vista da percepção térmica, conforme esta é relatada por indivíduos, o processo se inicia nos receptores térmicos localizados à superfície da pele (epiderme), os quais transmitem informações acerca da temperatura da superfície do corpo, que, por sua vez, mediam processos de regulação térmica (denominados mecanismos homeotérmicos). A sensação térmica percebida é responsável pelo comportamento termorregulador, como abrir janelas, ajustar o equipamento de climatização, alterar a vestimenta ou postura, procurar abrigar-se em locais mais confortáveis, entre outros, aumentando o alcance de medidas homeostáticas para além das reações fisiológicas (TOCHIHARA et al., 2012). Ao mesmo tempo em que o corpo experimenta tais estímulos, a percepção térmica é expressa por meio de categorias de estresse térmico no cérebro do indivíduo. Alguns autores analisam como se dá o processo de traduzir em palavras a sensação térmica experimentada (TOCHIHARA et al., 2012; LEE; TOCHIHARA, 2010; LEE et al., 2010). Uma dificuldade muitas vezes comentada nesses estudos se relaciona com a resposta de diferentes populações (com idiomas diferentes), sob condições climáticas e contextos diferentes, o que traz limitações a resultados mais conclusivos. O estudo relatado por Tochihara et al. (2012), por exemplo, no qual se comparam questões linguísticas nas escalas de sensação térmica para três grupos étnicos diversos (indonésio, inglês e japonês), mostrou diferenças tanto nas condições climáticas vigentes durante a aplicação do questionário quanto no efeito combinado da etnia, cultura e características climáticas de cada região.

Como referido por Andersen e Maibach (1979), pesquisas focadas em diferenças raciais ou na cor

${ }^{1} \mathrm{Na}$ língua inglesa, há diferenças entre "strain" (com efeitos no estresse físico, fisiológico) e "stress" (características indesejáveis do ambiente térmico, que, por sua vez, trarão alterações no estresse fisiológico) (PARSONS, 2014). da pele de indivíduos continuam escassas devido a aspectos emocionais envolvidos ao se temer que os resultados sirvam como base para preconceitos raciais de diversas formas. Conforme os autores citados há também uma dificuldade adicional em se isolarem fatores socioeconômicos e culturais inerentes à etnicidade de indivíduos de cor de pele diferentes.

A pele é o principal órgão controlador de fluxos térmicos entre o corpo e o meio circundante (ARENS; ZHANG, 2006). Em relação à sensação térmica, são os receptores da pele que participam do controle termorregulatório humano, influindo diretamente na sensação experimentada pelo indivíduo. A radiação solar e os ganhos por radiação de onda longa de superfícies do entorno podem aquecer a pele em função de sua cor e emissividade. A absortividade da pele varia em função de sua coloração, porém o efeito é mais evidente no comprimento de luz visível (0,4-0,7 $\mu \mathrm{m})$, com a pele clara tendo um coeficiente de absorção solar de cerca de 0,5 , e a cor negra, de 0,74 (HOUDAS; RING, 1982).

Narita et al. (2001) mostram que é na faixa visível que a sensibilidade da pele é maior. A energia radiante, quando chega à superfície da pele, pode ser absorvida ou refletida, havendo a partir disso um efeito térmico em sua temperatura superficial. Gonzalez (1983) mostra que, dentro de um intervalo de $60 \mathrm{~s}$ a partir da exposição da pele (ponto na testa) ao aquecimento solar, a diferença no acréscimo de temperatura entre cor da pele escura e clara é de praticamente o dobro para pessoas com cor mais escura. Interessantemente, embora haja diferenças entre a sensibilidade da pele em partes diversas do corpo, essas diferenças se reduzem com o aumento da radiação incidente: quanto maior o ganho térmico, menor se tornam as diferenças intracorporais.

Em situação de calor, o efeito das glândulas sudoríparas é considerado como tendo poucas variações entre indivíduos de colorações de pele diferentes, sendo um fator complicador nos estudos de campo uma comparação direta da sudorese de diferentes grupos, uma vez que pode haver diferenças genéticas e/ou de adaptação ao ambiente climático (JABLONSKI, 2004). Entretanto, Taylor (2006) demonstra que processos homeotérmicos ocorrem de forma diferenciada para indivíduos com diferentes características raciais. Na presença de calor, os processos adaptativos fisiológicos envolvem a redução da temperatura interna do corpo, a redução da frequência cardíaca e o aumento na produção de suor. Taylor indica que as diferenças mais 
evidentes são quanto a esse último processo. Porém, pesquisas quanto à quantidade e ativação de glândulas sudoríparas para indivíduos com diferentes etnias trazem, resumidamente, dois fatores de influência: aclimatação nos primeiros anos de vida do indivíduo e diferenças morfológicas entre indivíduos (área de superfície do corpo).

Particularmente quanto a diferenças na absortividade da pele, assume-se como hipótese, na presente pesquisa, que o fator cor da pele traz consequências na percepção térmica experimentada e relatada por indivíduos, e de forma acentuada sob condições de calor e na presença de ganhos por radiação solar no espaço aberto.

Especificamente no caso do Rio de Janeiro, a grande variação quanto à cor da pele dos indivíduos é marcante e representativa da população local. Segundo o IBGE, tem-se para essa cidade a distribuição de cor/raça (IBGE, 2012) conforme a Tabela 1, segundo as cinco classes de cor/raça adotadas, quais sejam: branca, preta, amarela, parda e indígena. $\mathrm{O}$ total não fecha os $100 \%$, pois há um percentual ínfimo de pessoas sem denominação racial, de $0,01 \%$; a população residente foi de 15.989.929 para o ano de levantamento de 2010 .

\section{Objetivo}

O objetivo do presente estudo é analisar o efeito da cor da pele na percepção térmica conforme relatada por transeuntes em levantamentos de campo nas ruas do centro do Rio de Janeiro. Avalia-se a relevância de se levarem em conta aspectos demográficos (neste caso, a origem étnica dos entrevistados) em levantamentos de percepção térmica em espaços abertos.

\section{Método}

O método adotado envolveu pesquisa de campo quanto à percepção térmica de transeuntes, e simultaneamente foi realizada coleta de dados meteorológicos.

O levantamento de dados foi realizado mediante questionários estruturados com base na ISO 10551 (INTERNATIONAL..., 1995), acerca da percepção térmica de transeuntes ao ar livre, aplicados à população local. A população de estudo foi definida como sendo aquela que trabalha e circula no centro da cidade do Rio de Janeiro e reside na área do Grande Rio e Grande Niterói, dadas as semelhanças climáticas das duas regiões vizinhas.

\section{Área de estudo e localização dos pontos de medição}

De acordo com a classificação de Köppen-Geiger a região da cidade do Rio de Janeiro (22 $2^{\circ} 4^{\prime} 10^{\prime \prime S}$, $\left.43^{\circ} 12^{\prime} 27^{\prime \prime} \mathrm{W}\right)$ é caracterizada por um clima tropical com chuvas de verão (Aw). A região do centro da cidade do Rio de Janeiro possui uma área total de 572,31 ha (em 2003) e uma população total de 41.142 pessoas, segundo dados do IBGE (IBGE, 2012). A região é delimitada pelos bairros de Catumbi, Cidade Nova, Estácio, Gamboa, Glória, Praça da Bandeira, Santo Cristo e Saúde.

Apesar de ter pequena população residente comparativamente a outros bairros da cidade, a região se caracteriza por ter uma grande população diurna, envolvida com as atividades de negócios e comércio, e, ainda, grande quantidade de pessoas que circulam pela região, de passagem para outros bairros.

Todos os pontos nos quais foram realizadas medições microclimáticas e entrevistas com transeuntes se localizam em ruas de pedestres com acesso a veículos permitido apenas para serviços (entregas e retiradas de mercadorias e outras atividades relacionadas à manutenção do comércio, escritórios, empresas, etc.). A predefinição dos pontos de monitoramento partiu da avaliação do entorno de cada ponto cogitado para as medições, sendo determinado na ocasião também o fator de visão do céu (FVC) por meio de câmera com lente olho de peixe. Em resumo, têm-se: os pontos 1 e 4 em praças secas com baixa presença de vegetação; o ponto 2 em rua densamente vegetada; o ponto 3 em cânion urbano sem vegetação significativa; os pontos 5 e 7 em cânions urbanos profundos sem vegetação; e o ponto 6 em cruzamento de ruas de pedestres. Os valores de FVC variaram entre $4 \%$ e $45 \%$, apresentando-se suficientemente diferenciados para representar a diversidade de situações urbanas existentes no centro do Rio de Janeiro. Dessa forma, foram realizadas 18 campanhas de monitoramento no total durante os períodos indicados na Tabela 2 e conforme a localização de cada ponto, representada na Figura 1 .

\section{Pesquisa de campo: medição de dados meteorológicos e aplicação de questionários}

A coleta de dados foi realizada com uma estação meteorológica Davis Vantage Pro2, equipamento wireless constituído por duas partes: conjunto de sensores e console digital (datalogger), responsável pelo armazenamento de dados. O 
conjunto de sensores é composto de sensor de temperatura e umidade do ar, anemômetro de copo com pá de vento, piranômetro de silício e coletor de água de chuva. Para a obtenção da temperatura radiante média, adotou-se uma esfera de PVC com diâmetro aproximado de $110 \mathrm{~mm}$, pintada na cor cinza, na qual se inseriu um medidor de temperatura (Tinytag TGP-4500). As características do conjunto de sensores utilizados estão indicadas na Tabela 3.

Tabela 1 - Distribuição da população residente por cor ou raça para a cidade do Rio de Janeiro

Fonte: IBGE (2010).

\begin{tabular}{c|c}
\hline Classe & Percentual \\
\hline Branca & 47,42 \\
Preta & 12,37 \\
Amarela & 0,77 \\
Parda & 39,33 \\
Indígena & 0,10 \\
\hline
\end{tabular}

Figura 1 - Mapa com o trecho de pedestres analisado e os sete pontos de medição

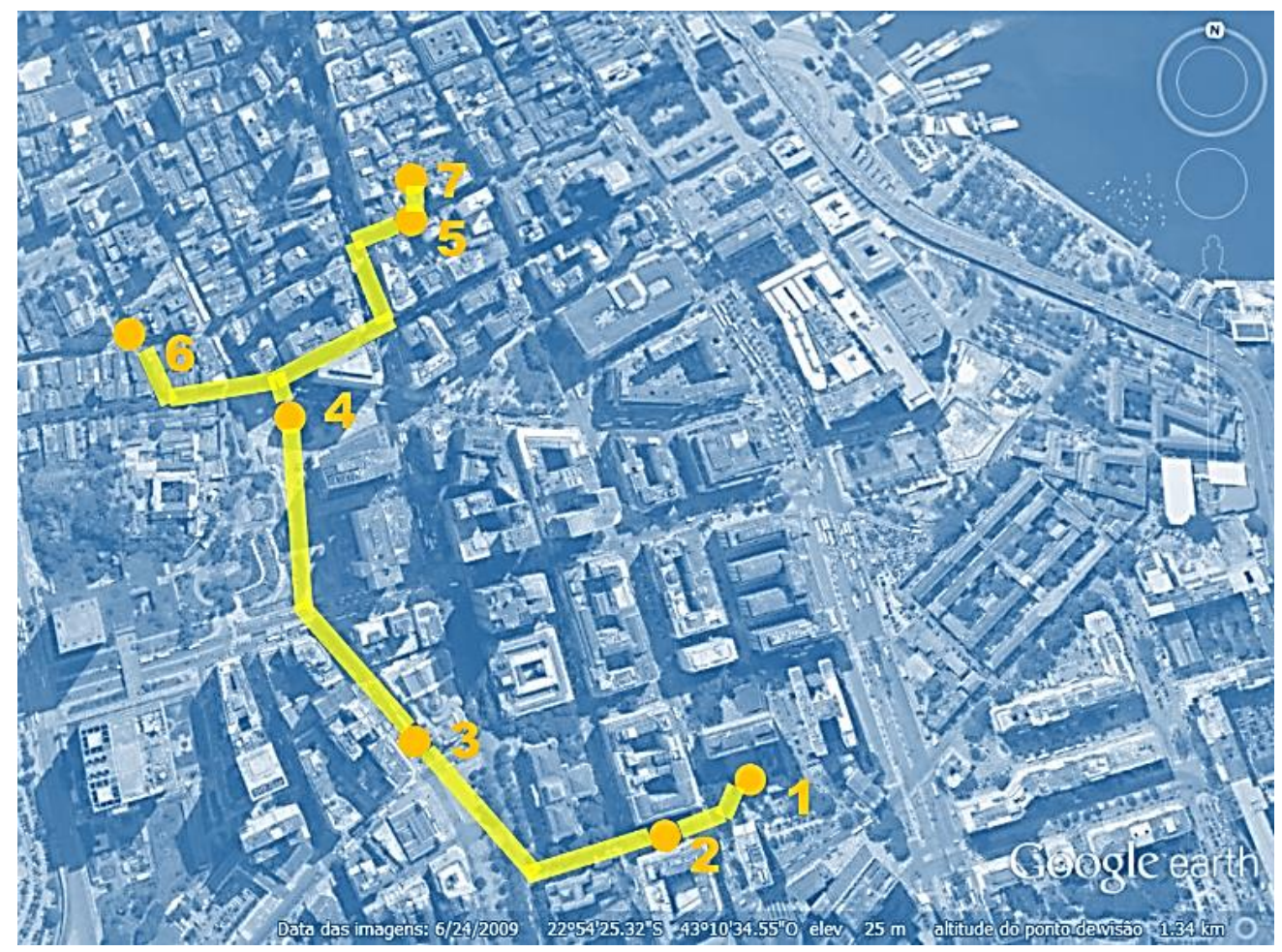

Tabela 2 - Pontos de monitoramento, FVC, dias monitorados e número de respondentes (n)

\begin{tabular}{c|c|c|c}
\hline Ponto & FVC & Dia do ano & n \\
\hline Ponto 1 & 0,27 & $7^{\mathrm{a}}, 354^{\mathrm{b}}$ & 134 \\
Ponto 2 & 0,29 & $12^{\mathrm{a}}, 297^{\mathrm{c}}, 354^{\mathrm{b}}$ & 178 \\
Ponto 3 & 0,27 & $9^{\mathrm{a}}, 290^{\mathrm{c}}, 303^{\mathrm{c}}, 355^{\mathrm{b}}$ & 213 \\
Ponto 4 & 0,45 & $8^{\mathrm{a}}, 15^{\mathrm{b}}, 355^{\mathrm{b}}$ & 161 \\
Ponto 5 & 0,11 & $352^{\mathrm{b}}, 354^{\mathrm{c}}$ & 112 \\
Ponto 6 & 0,36 & $7^{\mathrm{d}}, 353^{\mathrm{d}}, 359^{\mathrm{c}}$ & 156 \\
Ponto 7 & 0,04 & $352^{\mathrm{b}}$ & 31 \\
\hline
\end{tabular}

Nota: ${ }^{\mathrm{a}}(2015),{ }^{\mathrm{b}}(2013),{ }^{\mathrm{c}}(2012) \mathrm{e}^{\mathrm{d}}(2014)$. 
O posicionamento do conjunto de sensores atendeu às recomendações da ISO 7726 (INTERNATIONAL..., 1998), sendo, portanto, os sensores de temperatura do ar, de umidade relativa e o termômetro de globo fixados à altura de 1,10 m. O piranômetro foi fixado a $1,40 \mathrm{~m}$, e o anemômetro, a $1,50 \mathrm{~m}$, de modo que os dados coletados estivessem o mais próximo possível do nível do pedestre/entrevistado. A temperatura radiante média (TRM) foi calculada a partir dos dados de temperatura (sensor encapsulado), temperatura do ar e velocidade do ar medidos em cada ponto, sendo utilizada a equação para convecção forçada, de acordo com a ISO 7726 (INTERNATIONAL..., 1998) (Equação 1). A emissividade da esfera de PVC cinza foi assumida como 0,95 , para corpos cinzas. A gravação dos dados ocorreu a cada $5 \mathrm{~s}$, sendo a média composta para cada minuto.

$\operatorname{Trm}=\left\{(\mathrm{Tg}+273)^{4}+\left[\left(1,1 \times 10^{8} \times \mathrm{Va}^{0,6}\right) /(\mathcal{E g ~ x}\right.\right.$ $\left.\left.\left.\mathrm{D}^{0,4}\right)\right] \times(\mathrm{Tg}-\mathrm{Ta})\right\}^{1 / 4}-273 \quad$ Eq. 1

Onde:

Trm = temperatura radiante média, em ${ }^{\circ} \mathrm{C}$;

$\mathrm{Tg}=$ temperatura de globo, $\mathrm{em}^{\circ} \mathrm{C}$;

va $=$ velocidade do vento, $\mathrm{em} \mathrm{m} / \mathrm{s}$;

$\varepsilon g=$ emissividade do globo (adimensional);
$\mathrm{D}=$ diâmetro do globo, em metros; e

$\mathrm{Ta}=$ temperatura do ar, em ${ }^{\circ} \mathrm{C}$.

O monitoramento das variáveis microclimáticas foi realizado no período diurno (das $10 \mathrm{~h}$ às $15 \mathrm{~h}$ ), com aplicação simultânea de questionários de sensação e preferência térmica. Os dados climáticos foram registrados na estação meteorológica, sendo posteriormente associados aos momentos de cada entrevista. O questionário foi definido a partir da ISO 10551 (INTERNATIONAL..., 1995), e entre as questões estão incluídas informações demográficas, questões relacionadas ao processo de aclimatação e histórico térmico do entrevistado (tempo de residência na cidade, uso ou não de arcondicionado em casa ou no trabalho, tempo de permanência no espaço externo), assim como informações acerca da vestimenta utilizada, adotando-se uma tabela resumo de trajes típicos, conforme apresentado no Anexo A da ISO 9920 (INTERNATIONAL..., 2007). Para avaliar a percepção térmica (sensação e preferência), foi utilizada a escala simétrica de 7 pontos. A sensação térmica ("Como você se sente neste exato momento?") e a preferência térmica ("Por favor, relate como você preferiria estar se sentindo") foram categorizadas conforme a Tabela 4 , abaixo.

Tabela 3 - Características dos sensores utilizados nas medições

\begin{tabular}{l|c|c|c}
\hline \multicolumn{1}{c|}{ Sensor } & $\begin{array}{c}\text { Resolução por } \\
\text { unidade de medida }\end{array}$ & $\begin{array}{c}\text { Intervalo de } \\
\text { medição }\end{array}$ & Precisão \\
\hline Temperatura do ar & $0,1{ }^{\circ} \mathrm{C}$ & $-40{ }^{\circ} \mathrm{C} \mathrm{a}+65^{\circ} \mathrm{C}$ & $\begin{array}{c} \pm 0,5^{\circ} \mathrm{C} \text { acima de } \\
20{ }^{\circ} \mathrm{F}\left(-7^{\circ} \mathrm{C}\right)\end{array}$ \\
\hline Umidade do ar & $1 \%$ & $1 \%$ a $100 \%$ & $\begin{array}{c} \pm 3 \%(0-90 \%), \pm 4 \% \\
(90-100 \%)\end{array}$ \\
\hline Velocidade do ar & $0,4 \mathrm{~m} / \mathrm{s}$ & $1 \mathrm{a} 80 \mathrm{~m} / \mathrm{s}$ & $\pm 1 \mathrm{~m} / \mathrm{s}$ \\
\hline Direção do vento & $22,5^{\circ}$ & $0-360^{\circ}$ & $\pm 3^{\circ}$ \\
\hline Radiação solar & $1 \mathrm{~W} / \mathrm{m}^{2}$ & $0 \mathrm{a} 1.800 \mathrm{~W} / \mathrm{m}^{2}$ & $\pm 5 \%$ \\
\hline $\begin{array}{l}\text { Temperatura do ar (Tinytag TGP- } \\
\text { 4500) }\end{array}$ & $0,01{ }^{\circ} \mathrm{C}$ & $-25{ }^{\circ} \mathrm{C} \mathrm{a}+85^{\circ} \mathrm{C}$ & $\pm 0,45^{\circ} \mathrm{C}$ \\
\hline
\end{tabular}

Tabela 4 - Pontuação para sensação e preferência térmicas

\begin{tabular}{c|c|c|c}
\hline Pontuação & Resposta & Pontuação & Resposta \\
\hline$(-3)$ & "muito frio" & $(+3)$ & "muito mais calor" \\
$(-2)$ & "frio" & $(+2)$ & "mais calor" \\
$(-1)$ & "pouco frio" & $(+1)$ & "um pouco mais de calor" \\
$(0)$ & "neutro" & $(0)$ & "sem mudanças" \\
$(+1)$ & "pouco calor" & $(-1)$ & "um pouco mais frio" \\
$(+2)$ & "calor" & $(-2)$ & "mais frio" \\
$(+3)$ & "muito calor" & $(-3)$ & "muito mais frio" \\
\hline
\end{tabular}


No caso da taxa metabólica, foi assumido um valor fixo de 2,3 Met, o que corresponde a uma pessoa caminhando a uma velocidade de $4 \mathrm{~km} / \mathrm{h}^{2}$, situação considerada como referência para a taxa metabólica do índice de conforto UTCI adotado (ver seção seguinte).

No que diz respeito à autoclassificação quanto à cor da pele de cada entrevistado e juntamente com as questões demográficas (sexo, idade, altura, peso), na Parte I do questionário incluiu-se a questão "Como você classifica sua cor da pele?", sugerindo-se para o respondente as cinco classes adotadas pelo IBGE (cf. Tabela 1).

As variáveis tempo de residência e tempo de permanência no espaço aberto foram consideradas como critério de exclusão quando inferiores a 6 meses e 15 min respectivamente. Seis meses de residência na cidade e cercanias correspondem ao ciclo de insolação para determinada latitude, englobando, no caso deste estudo, estação fria e meia-estação. O limite de $15 \mathrm{~min}$ foi adequado a partir do tempo mínimo recomendado pela ANSI/ASHRAE 55 (AMERICAN..., 2004) para estudos de conforto em espaços internos. Foram excluídas pessoas com pesos extremos (máximo e mínimo) e crianças.

\section{Índice térmico adotado}

A utilização de índices térmicos em espaços abertos se justifica na presente pesquisa porque tais índices integram as variáveis microclimáticas medidas nos diversos pontos de monitoramento simultaneamente à aplicação dos questionários. Adotou-se um índice de uso corrente nos campos da climatologia urbana e biometeorologia humana, o Universal Thermal Climate Index (UTCI). Tal índice foi elaborado com base no modelo termorregulatório multinodal desenvolvido por Fiala et al. (2012) e é baseado em uma temperatura equivalente em ambiente de referência. $O$ índice estima uma resposta fisiológica dinâmica multidimensional (temperatura corporal, taxa de sudorese, temperatura da pele, etc., em diferentes tempos de exposição) para dada combinação das variáveis climáticas (vento, radiação, umidade e temperatura do ar). No Brasil já há alguns trabalhos que utilizam o índice UTCI para a predição da sensação térmica no espaço aberto (ROSSI; KRÜGER; BRÖDE, 2012; MONTEIRO; ALUCCI, 2012; NINCE et al., 2013). Como elemento adicional para análise, utilizou-se a variável Dynamic Thermal Sensation (DTS), a qual representa a sensação térmica na mesma escala de sete pontos utilizada nos levantamentos.
A DTS é calculada diretamente a partir de dados de UTCI e expressa a sensação térmica estimada.

\section{Amostra obtida}

A amostra resultante analisada é composta de dados de primavera/verão, com 985 entrevistados, sendo $61 \%$ do sexo masculino e $39 \%$ do sexo feminino, com idade variando entre 13 e 87 anos. Quanto às variações climáticas encontradas ao longo dos diversos dias de medição, a Tabela 5 mostra as faixas de variação das variáveis monitoradas.

A amostra é constituída de 561 pessoas que declararam ter cor da pele branca, 277 de cor parda, 139 de cor preta, com ainda 5 indivíduos que se declararam indígenas e 3 asiáticos (cor amarela), perfazendo o total de 985 transeuntes. Comparativamente à distribuição demográfica levantada para o IBGE, embora haja o mesmo ordenamento por classes raciais (população de cor de pele branca mais numerosa em relação às demais), há notadamente diferenças na amostra levantada quanto à distribuição percentual por cor da pele (Figura 2).

\section{Procedimento de análise}

A análise dos dados obtidos envolveu as seguintes etapas:

(a) agrupamento de dados segundo categorias de cor/raça (IBGE, 2012);

(b) agrupamento de votos de sensação térmica por variações de $1^{\circ}$ na escala do índice térmico adotado (UTCI);

(c) comparações por análise de regressão linear entre dados agrupados (bins) para diferentes categorias de cor/raça (IBGE, 2012);

(d) comparações por classe de estresse térmico do índice UTCI dos percentuais de pessoas em situação declarada de frio, conforto e calor, para diferentes categorias étnicas; e

(e) ajuste da amostra para a composição demográfica observada para a cidade do Rio de Janeiro - adotou-se um procedimento de randomização da amostra para as três maiores categorias observadas na amostra (cor da pele branca, parda e preta). A randomização teve por objetivo aproximar a distribuição demográfica da amostra segundo o aspecto de cor da pele à do IBGE para a cidade do Rio de Janeiro, de modo a que se pudesse avaliar a percepção térmica de uma amostra mais representativa da população local no espaço aberto. 
Tabela 5 - Faixas de variação dos dias monitorados

\begin{tabular}{|c|c|c|c|c|c|}
\hline & $\begin{array}{l}\text { Temperatura do } \\
\operatorname{ar}\left(\mathbf{T}_{\mathrm{a}} \mathrm{em}^{\circ} \mathrm{C}\right)\end{array}$ & $\begin{array}{c}\text { Umidade } \\
\text { relativa (UR } \\
\text { em \%) }\end{array}$ & $\begin{array}{l}\text { Velocidade } \\
\text { do ar (v } \\
\text { em } \mathbf{m} / \mathbf{s})\end{array}$ & $\begin{array}{c}\text { Irradiância solar } \\
\text { global e refletida } \\
\left(\operatorname{Ig~em~W/m^{2}}\right)\end{array}$ & $\begin{array}{c}\mathbf{T}_{\text {mrt }}-\text { Temperatura } \\
\text { radiante média } \\
\left(\text { TRM em }^{\circ} \mathrm{C}\right)\end{array}$ \\
\hline $\begin{array}{l}\text { Faixa de } \\
\text { variação }\end{array}$ & $24,9-36,8$ & $37-82$ & $0-2,7$ & $21-1218$ & $13,1-76,4$ \\
\hline
\end{tabular}

Figura 2 - (a) Distribuição da população residente por cor da pele ou raça para a cidade do Rio de Janeiro (IBGE, 2012); e (b) composição da amostra segundo as mesmas classes do IBGE

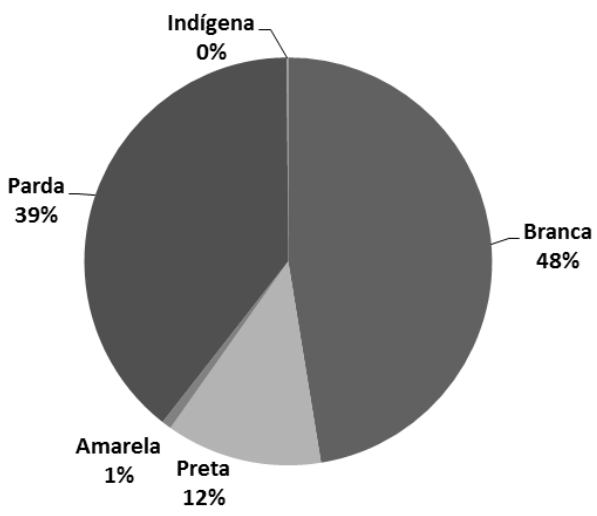

(a)

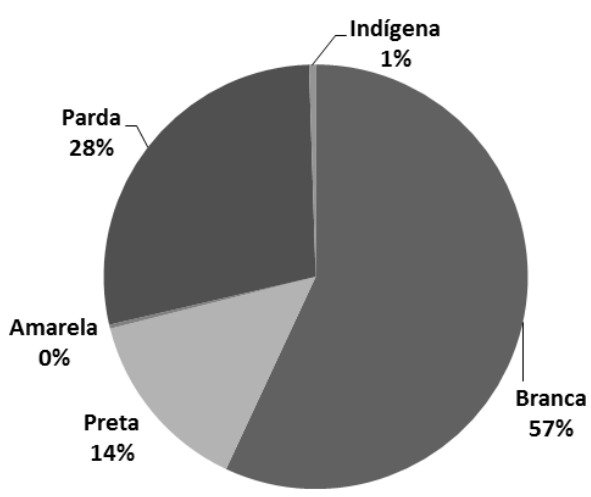

(b)

\section{Resultados encontrados}

Quanto aos votos de sensação térmica, conforme reportados pelos entrevistados, foram geradas equações de regressão para a obtenção da sensação térmica estimada a partir de valores na escala UTCI para os grupos de transeuntes classificados como tendo cor da pele branca, parda e preta ${ }^{3}$. Os dados de campo foram agrupados em bins, garantindo-se que cada bin contivesse a média de no mínimo 5 votos de percepção térmica e de forma que a comparação se desse na mesma faixa de variação de UTCI (no caso, com UTCI variando entre $28{ }^{\circ} \mathrm{C}$ e $\left.42{ }^{\circ} \mathrm{C}\right)$. Tal agrupamento de respostas de sensação térmica por variação de um grau na escala de determinado índice térmico foi realizado conforme procedimento sugerido por de Dear e Fountain (1994) e utilizado em estudos semelhantes (DEUBLE; DE DEAR, 2012; KRÜGER et al., 2012; LIN; LIN; HWANG, 2013; NDETTO; MATZARAKIS, 2013; DE DEAR et al., 2014; NDETTO; MATZARAKIS, 2015). A partir dos dados agrupados, gera-se uma linha de tendência linear para cada série (cf. Figuras 3 e 4).

A amostra resultante de indivíduos após a aplicação do procedimento de agrupamento descrito e com a exclusão de pessoas de cor da pele amarela e indígena reduziu-se em $4 \%$, totalizando 942 pessoas.

${ }^{3}$ Os percentuais de pessoas com cor da pele amarela e indígena foram considerados desprezíveis (total inferior a 1\% da amostra obtida).
Para a condição inicial de calor, com voto de sensação térmica equivalente a "pouco calor" (valor +1$)$, situação inicial de estresse térmico, as temperaturas na escala UTCI diferem em $2,4{ }^{\circ} \mathrm{C}$ na escala UTCI entre pessoas de cor da pele preta e branca, sendo de $28,3{ }^{\circ} \mathrm{C}$ e $30,7{ }^{\circ} \mathrm{C}$ respectivamente, conforme a Figura 3. Nota-se menor dispersão entre os votos de sensação térmica de pessoas de cor da pele branca do que nos demais grupos. $\mathrm{O}$ valor $p$ encontrado para a diferença entre os grupos mais díspares (cor da pele branca versus preta) mostrou, entretanto, baixa significância estatística $(\mathrm{p}=0,4)$.

Em termos de sensação térmica predita (valor de DTS), calculada juntamente com o UTCI, a diferença em termos de voto de sensação térmica reportada é de 0,3 (Figura 4), novamente apontando para uma maior tolerância ao calor no caso de pessoas de cor da pele branca. O gráfico sugere também a necessidade de calibração do índice DTS para as condições de sensação térmica reportadas; o coeficiente angular de ambas as curvas de tendência mostra maior tolerância a condições de calor em situação real, independentemente da categoria de cor da pele, do que os valores preditos em situação de extremo calor. Para DTS=2, por exemplo, encontram-se os votos de sensação térmica 1,8 e 1,6 para os grupos de pessoas de cor da pele preta e branca respectivamente; para $\mathrm{DTS}=3$, os votos de sensação térmica são 2,5 e 2,4 respectivamente. 
Figura 3 - Dados na escala UTCI $\left({ }^{\circ} \mathrm{C}\right)$ versus votos de sensação térmica (VST), agrupados por raça/cor da pele - a linha demarcada representa a diferença entre os grupos mais díspares ('branca' e 'preta') em termos de valores UTCI para "pouco calor"

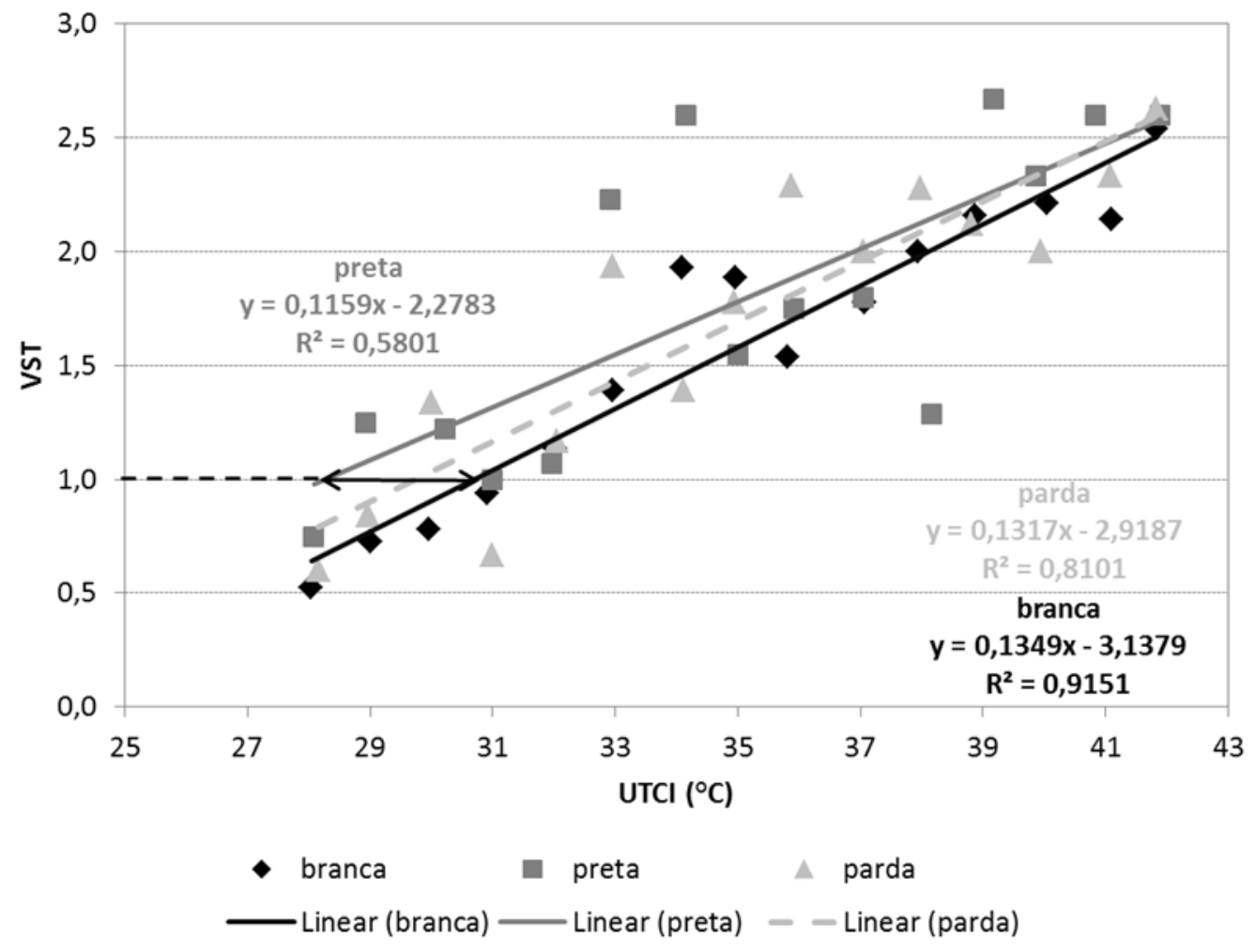

Figura 4 - Dados de sensação térmica dinâmica (escala DTS) versus votos de sensação térmica (VST), agrupados por agrupados por raça/cor da pele - a linha demarcada representa a diferença entre os grupos em termos de valores de sensação térmica para "pouco calor" na escala DTS

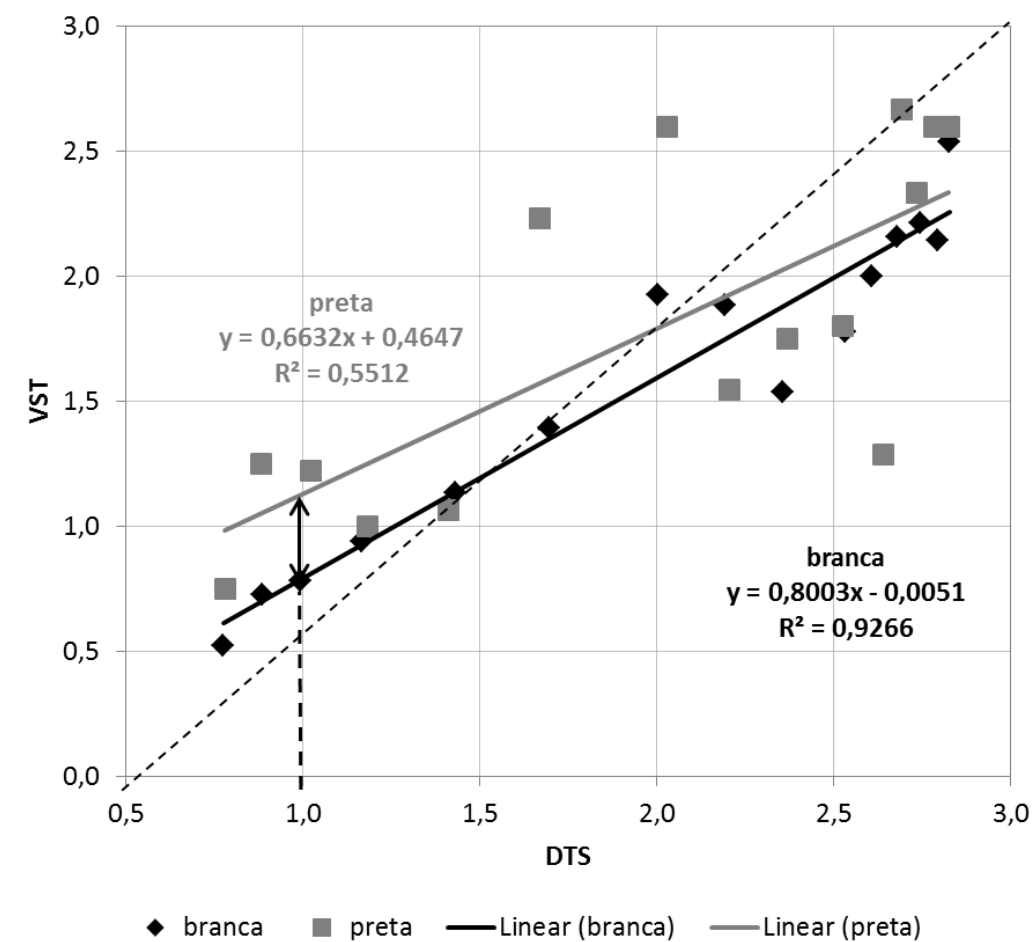

90 Krüger, E. L.; Drach, P. R. C. 
Classificando-se as respostas segundo as classes de estresse térmico de UTCI (Tabela 6), obtiveram-se os percentuais dos votos de sensação térmica conforme declarados pelos transeuntes, por classe de estresse térmico. Adotam-se como padrão os votos de sensação térmica reportados de -3 e -2 como correspondendo a "frio", os votos entre -1 e +1 como sendo "conforto", e os votos +2 e +3 como sendo "calor". Nota-se uma pequena diferença em termos de menor tolerância ao calor para pessoas de cor da pele preta em relação àqueles de cor da pele branca (Figuras 5 e 6). $\mathrm{O}$ percentual de pessoas em situação reportada de calor, com votos de sensação térmica superiores a +1 , aumenta em $11 \%$ no caso de pessoas de cor da pele preta, enquanto a sensação de conforto desaparece na mesma proporção no caso da maior classe de estresse térmico atingida para o UTCI ("estresse térmico muito forte").
Quanto à preferência térmica, nota-se uma curva mais acentuada para pessoas de cor da pele branca, com um coeficiente de correlação quase tão alto quanto no caso dos votos de sensação térmica. A linha de tendência para o grupo de cor da pele preta, embora com pouca aderência (Figura 7), mostra uma tendência de menor variação quanto à preferência térmica para aumentos graduais no índice UTCI. Isso se traduz para uma maior indiferença térmica do segundo grupo para aumentos de estresse térmico por calor, conforme representados na escala UTCI. Os coeficientes angulares das duas curvas de tendência obtidas são visivelmente distintos. $\mathrm{O}$ valor $p$ encontrado para a diferença entre os grupos (cor da pele branca versus preta) mostrou significância estatística $(\mathrm{p}<0,05)$.

Tabela 6 - Categorias de estresse térmico para calor pelo índice UTCI

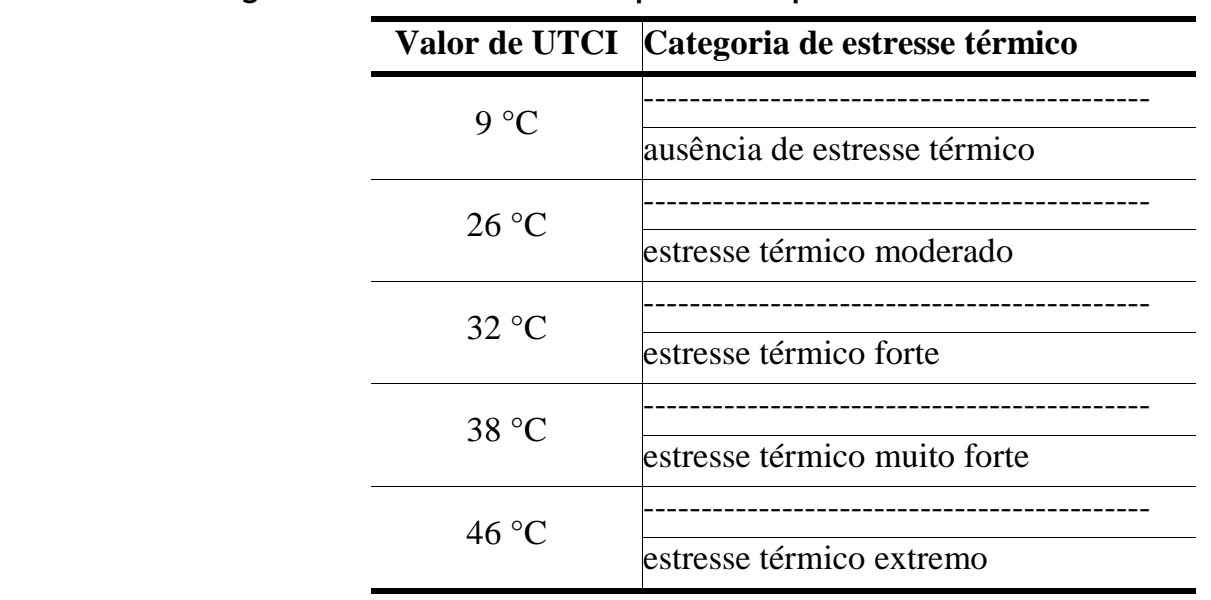

Fonte: conforme Bröde et al. (2012).

Figura 5 - Agrupamento dos votos de sensação térmica por classe de estresse térmico segundo o índice UTCI - grupo com cor da pele branca

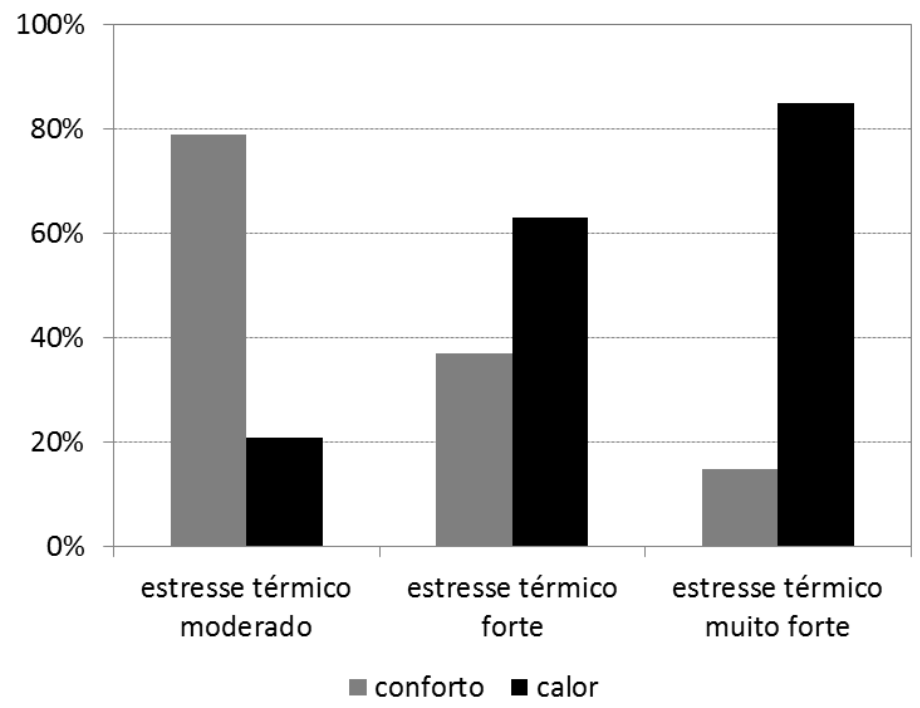


Figura 6 - Agrupamento dos votos de sensação térmica por classe de estresse térmico segundo o índice $\mathrm{UTCl}$ - grupo com cor da pele preta

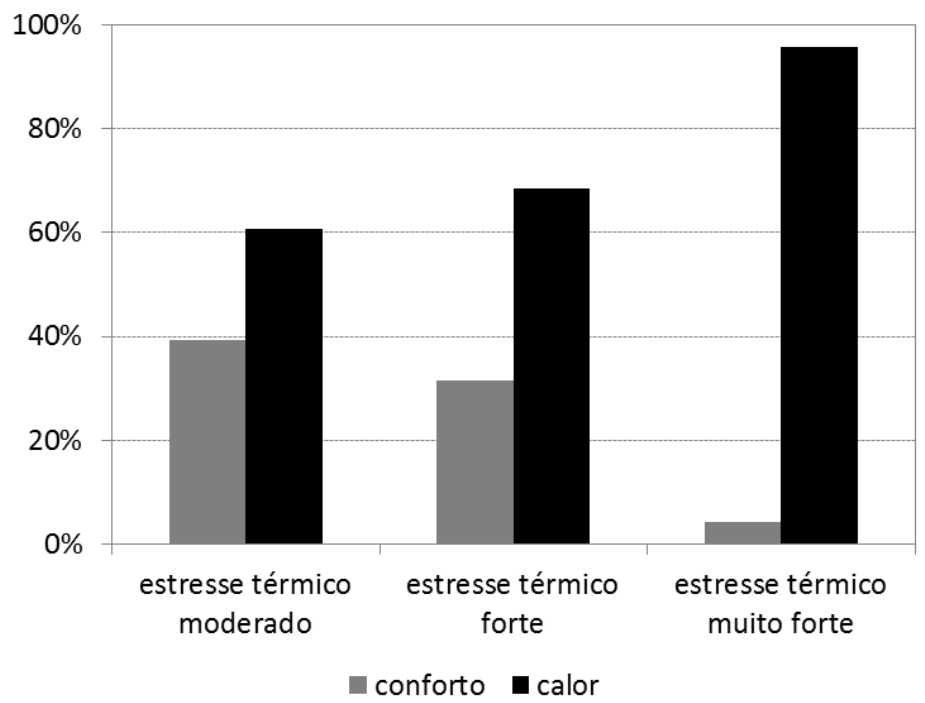

Figura 7 - Dados na escala UTCI $\left({ }^{\circ} \mathrm{C}\right)$ versus votos de preferência térmica, agrupados por cor da pele (cor da pele preta versus branca)

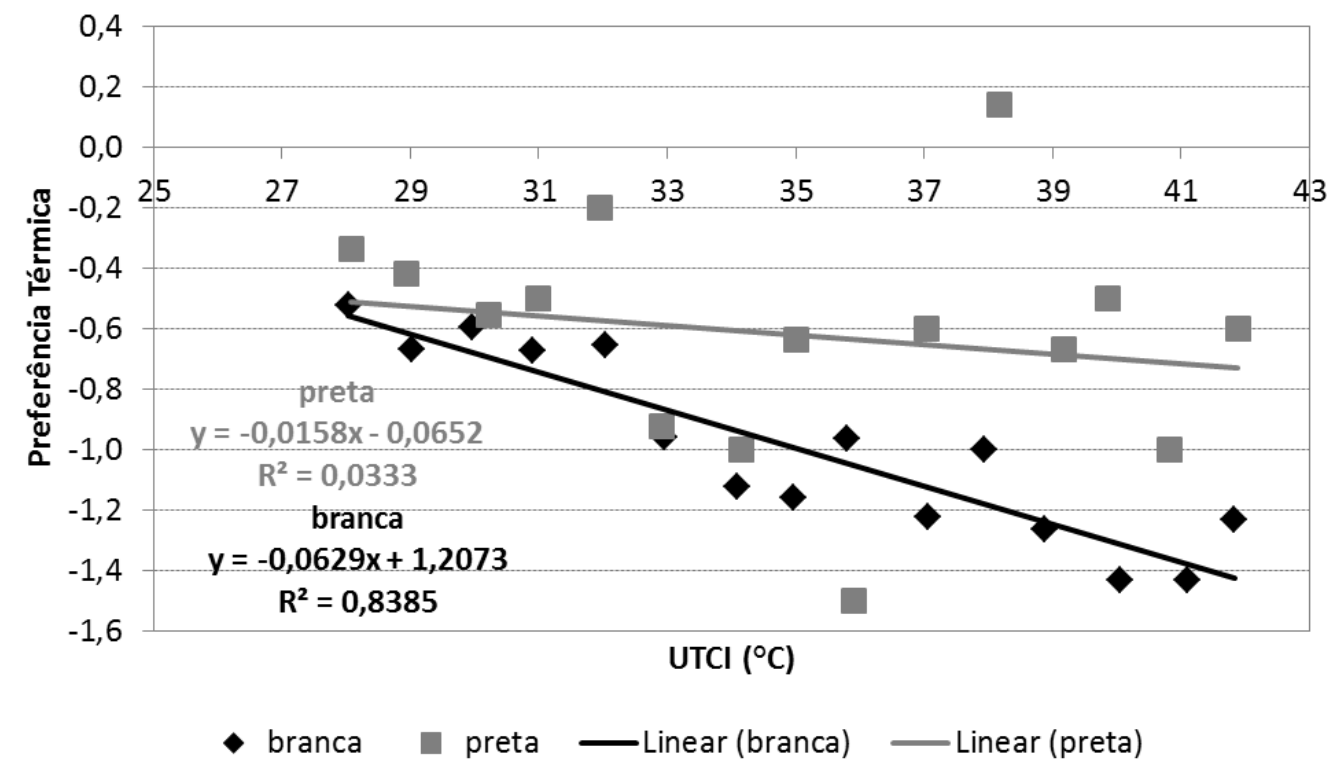

A linha de tendência para o agrupamento de indivíduos de cor negra, parda, indígena e asiáticos (categoria "não branca", n=417), com correlação significativa, mostra menor sensibilidade na preferência térmica para aumentos de UTCI (Figura 8), novamente representando maior insensibilidade da categoria "não branca" para aumentos no estresse térmico por calor. Para $\mathrm{UTCI}=38{ }^{\circ} \mathrm{C}$, valor inicial da faixa denominada "estresse térmico muito forte" (conf. Tabela 6), a variação em termos de votos de preferência térmica é semelhante à observada em termos de sensação térmica e da ordem de 0,3 voto. $O$ ponto de interseção entre as duas curvas de regressão da
Figura 6 se situa em torno da neutralidade térmica (preferência “ 0 ”, ou "sem mudanças"), em $\mathrm{UTCI}=21{ }^{\circ} \mathrm{C}$, caindo na região de "ausência de estresse térmico" da Tabela 6. Considerando-se o voto médio de "-1" para preferência térmica, equivalente à resposta "um pouco de frio", a diferença em termos de UTCI para se obter a mesma resposta (média) em termos de preferência, segundo as duas curvas de tendência, para brancos e não brancos, foi de cerca de $5{ }^{\circ} \mathrm{C}$ na escala UTCI. $\mathrm{O}$ valor $p$ encontrado para a diferença entre os grupos (cor da pele branca versus "não branca") mostrou significância estatística $(\mathrm{p}<0,05)$. 
Figura 8 - Dados na escala UTCI $\left({ }^{\circ} \mathrm{C}\right)$ versus votos de preferência térmica agrupados por cor da pele (cor da pele preta versus "não branca") - a área demarcada representa a faixa denominada "estresse térmico muito forte" para o índice UTCI

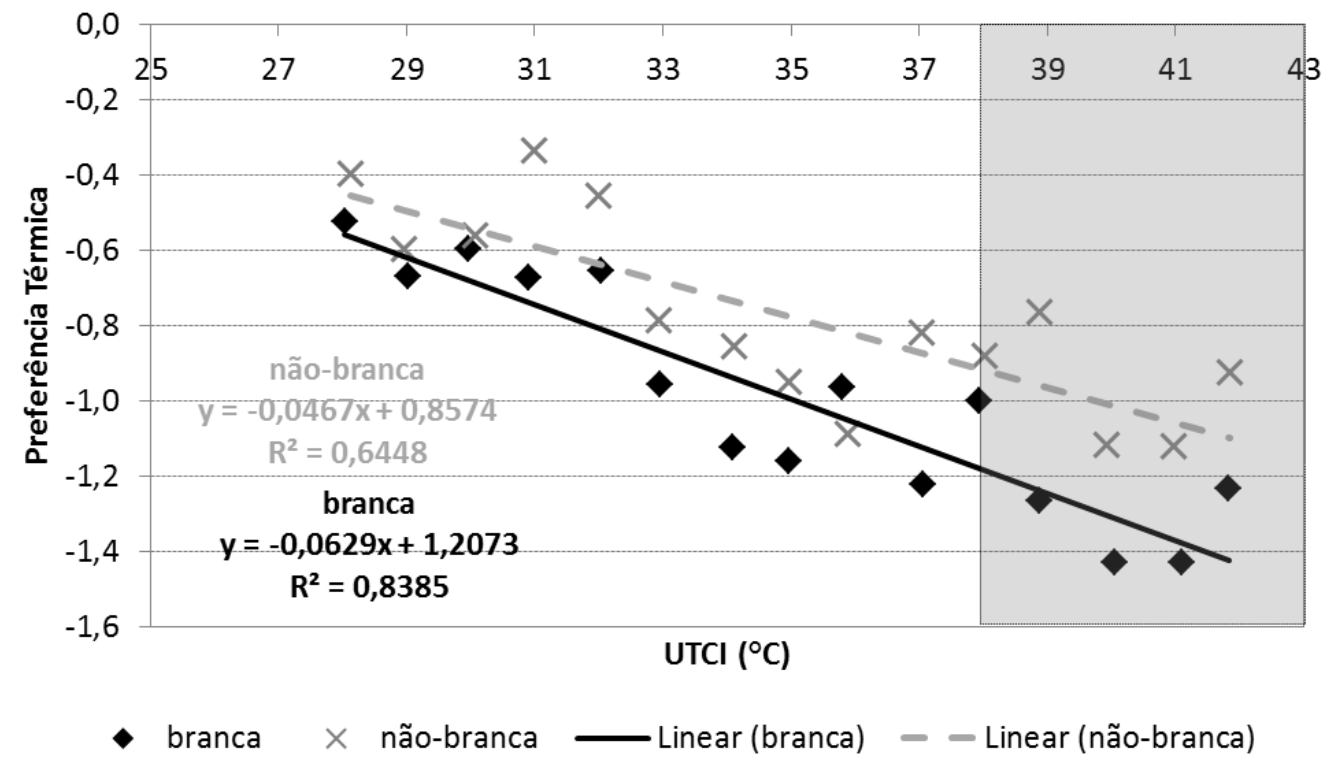

\section{Análise dos dados aleatoriamente agrupados para a distribuição demográfica do IBGE}

Como última etapa da pesquisa, randomizou-se a amostra de forma que ela melhor se adequasse à distribuição racial obtida pelo IBGE para a cidade do Rio de Janeiro (cf. Tabela 1). O processo de randomização foi realizado para os totais de cada grupo ao fixar-se o grupo onde se notou aumento percentual no ajuste à distribuição do IBGE. Dessa forma, o grupo de pessoas de cor da pele parda foi fixado como representando $39 \%$ da amostra ajustada (em substituição aos $28 \%$ de participação da Figura 2b), porém se mantendo $n=277$. Utilizou-se uma combinação de funções do Microsoft Excel (“Aleatório”, “Ordem”, "Procv”), adequando-se as quantidades de pessoas nos grupos de cor da pele branca e parda para alcançar os percentuais desejados. Dessa forma, obteve-se uma amostra com a seguinte distribuição: 5 pessoas nas categorias de cor da pele amarela e indígena (1\% do total); 87 indivíduos com cor da pele preta (12\% do total); e 334 indivíduos com cor da pele branca ( $47 \%$ do total). O total ajustado para a distribuição do IBGE resultou em 704 pessoas.
A curva de tendência resultante para a sensação térmica da amostra ajustada à distribuição por categorias étnicas é apresentada na Figura 9. Plotase adicionalmente a amostra original, previamente à redistribuição por categorias de cor da pele. Observa-se que, para a geração das linhas de tendência de cada amostra, retiraram-se as médias dos votos de sensação térmica reportados quando o número de respostas em cada variação de UTCI (bin) era inferior a 5 pessoas. Nota-se que a neutralidade térmica para o índice UTCI é obtida em $23,5{ }^{\circ} \mathrm{C}$ (escala UTCI), pela extensão da reta ou através de cálculo pela equação de regressão, situando-se dentro da faixa sugerida para o UTCI como sendo de "ausência de estresse térmico". O limiar do desconforto por calor (voto de sensação térmica +1) corresponde a $30,5{ }^{\circ} \mathrm{C}$ (escala UTCI). Tal limite situa-se próximo ao da amostra original, uma vez que nesse ponto se dá a interseção das duas curvas de regressão. Porém, a temperatura neutra, sem correção da amostra pela demografia local, é $1{ }^{\circ} \mathrm{C}$ inferior à da amostra corrigida. Há também alterações mais significativas na segunda e terceira classes de estresse térmico por calor $(+2$ $\mathrm{e}+3$ ), com a diferença entre ambas as amostras se acentuando em cerca de $1{ }^{\circ} \mathrm{C}$ e $2{ }^{\circ} \mathrm{C}$ respectivamente. 
Figura 9 - Dados na escala UTCI $\left({ }^{\circ} \mathrm{C}\right)$ versus votos de sensação térmica (VST) para a amostra ajustada à distribuição de cor da pele do IBGE (2010) para o Rio de Janeiro

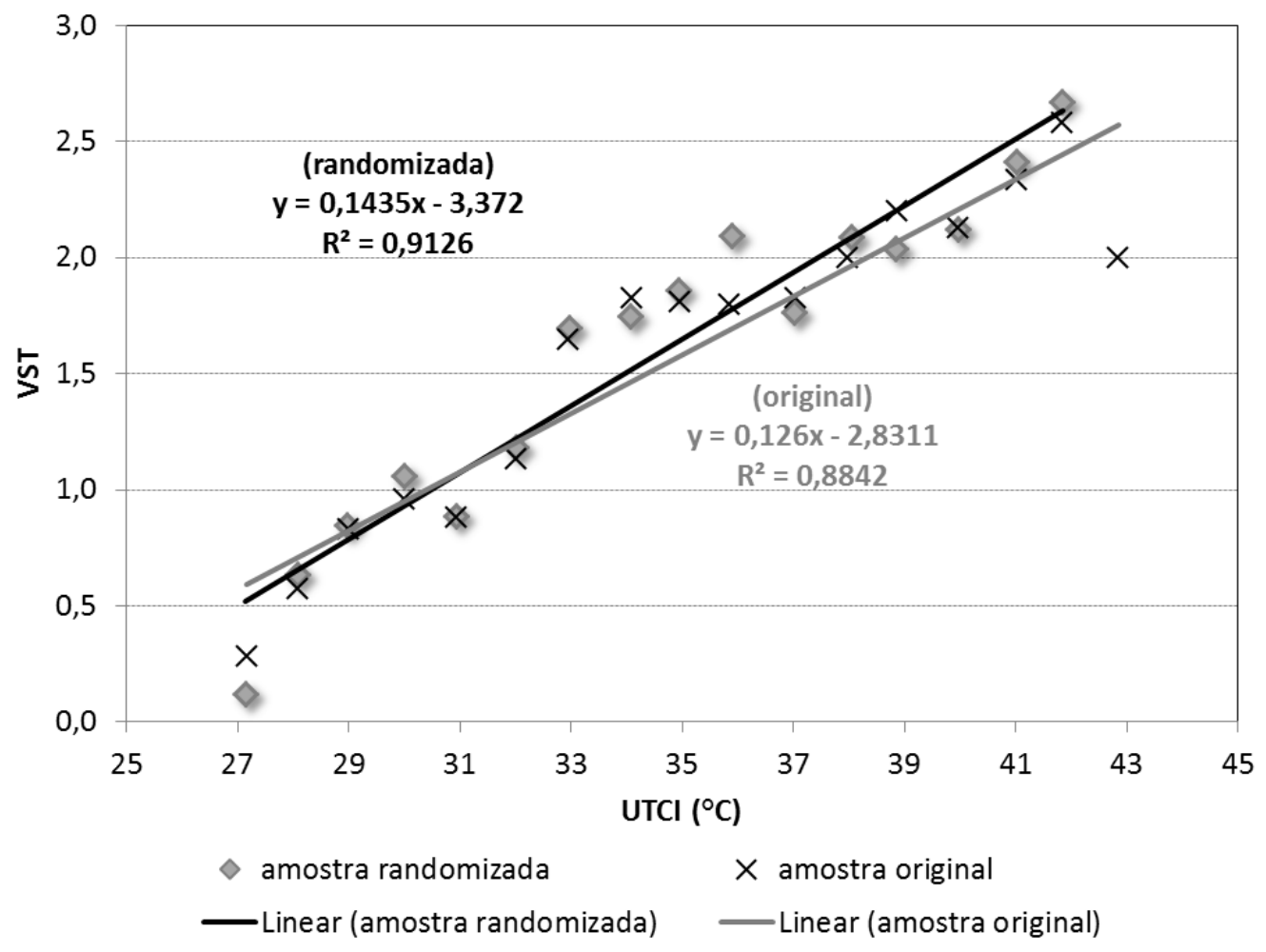

\section{Conclusões}

Comparando-se sensação e preferência térmicas sob o aspecto de coloração da pele, observa-se que, embora o grupo de pessoas de cor da pele branca tenha demonstrado maior tolerância ao estresse térmico por calor (em termos de sensação térmica média), quanto à preferência relatada este mostrou preferência mais acentuada por condições mais frias, de modo a neutralizar o desconforto. Notouse ainda quanto à preferência térmica maior indiferença térmica por parte do grupo de cor da pele preta e "não branca" para aumentos no estresse térmico ambiente. Observa-se que diferenças estatísticas foram obtidas apenas quanto à preferência térmica entre grupos de pessoas de cor da pele branca versus cor da pele preta ou "não branca" (representada por uma combinação dos grupos cor da pele parda, preta, indígena $\mathrm{e}$ amarela).

Uma limitação dos dados coletados quanto à cor da pele dos indivíduos se refere ao fato de ter sido usado o critério de autoclassificação de cada entrevistado segundo categorias raciais adotadas pelo IBGE. Métodos mais objetivos (como medidas de absortividade da superfície da pele) no levantamento dessa variável seriam recomendados para pesquisas futuras, assim como a classificação do entrevistado nas categorias do IBGE pelos próprios pesquisadores.
Como a superfície da pele sofre influências da vestimenta utilizada pelo entrevistado, seria interessante realizar levantamentos semelhantes em períodos mais frios do ano, quando há menor exposição da pele aos elementos do clima ou, ainda, em situações de maior exposição do corpo aos elementos do clima, como na praia. Uma continuidade da pesquisa de campo com a mesma instrumentação e segundo o mesmo protocolo de entrevistas está sendo programada para a cidade serrana de Petrópolis, RJ, onde o período de inverno mostra maior rigor térmico que na cidade do Rio de Janeiro, mantendo-se, porém, uma demografia semelhante em termos de cor da pele da população.

Os resultados obtidos mostram a complexidade de se realizarem levantamentos de percepção térmica em espaços abertos. Estudo anterior com a mesma amostra apontou diferenças significativas entre grupos de entrevistados que utilizavam ou não climatização artificial no trabalho e em casa (KRÜGER; DRACH, 2016). Aspectos demográficos como a cor da pele dos entrevistados elevam o grau de complexidade de tais levantamentos. Pode-se ainda apontar como possível linha de investigação o aspecto combinado de uso de climatização artificial e diferenciações de cor da pele entre indivíduos na amostra, investigando-se consequências para o uso 
de climatização artificial por diferentes extratos da população.

A discussão é oportuna e atual. Johansson et al. (2014), a partir de uma revisão de trabalhos em conforto em espaços abertos, apontam para a necessidade de se padronizarem os protocolos utilizados na realização de tais levantamentos. Recentemente foi criado um grupo de discussões, Outdoor Thermal Comfort Working Group, junto à International Association for Urban Climate (Iauc) com o objetivo de se discutirem procedimentos para estudos em conforto em espaços abertos, incluindo protocolos de monitoramento e levantamentos com os entrevistados.

\section{Referências}

AMERICAN SOCIETY OF HEATING, REFRIGERATING; AIR-CONDITIONING ENGINEERS. Standard 55: thermal environmental conditions for human occupancy. Atlanta, 2004.

ANDERSEN, K. E.; MAIBACH, H. I. Black and White Human Skin Differences. Journal of the American Academy of Dermatology, v. 1, p. 276-282, 1979.

ARENS, E.; ZHANG, H. The Skin's Role in Human Thermoregulation and Comfort. In: PAN, N.; GIBSON, P. (Eds.). Thermal and Moisture Transport in Fibrous Materials. Woodhead: [s.n.], 2006.

BRÖDE, P. et al. Deriving the Operational Procedure for the Universal Thermal Climate Index UTCI. International Journal of Biometeorology, v. 56, n. 3, p. 481-494, 2012.

DE DEAR, R. et al. Summertime Thermal Comfort in Australian School Classrooms. In: WINDSOR CONFERENCE: COUNTING THE COST OF COMFORT IN A CHANGING WORLD, 8., Cumberland Lodge, 2014. Proceedings... Cumberland Lodge, 2014.

DE DEAR, R. J.; FOUNTAIN, M. E. Cover Feature: thermal comfort in air-conditioned office buildings in the tropics. Journal of the Australian Institute of Refrigerating, Air-Conditioning and Heating, v. 48, n. 9, p. 14-30, 1994.

DEUBLE, M. P.; DE DEAR, R. Mixed-Mode Buildings: a double standard in occupants' comfort expectations related. Building and Environment, v. 54, n. 8, p. 53-60, 2012.

FIALA, D. et al. UTCI: fiala multi-node model of human heat transfer and temperature regulation. International Journal of Biometeorology, v. 56, n. 3, p. 429-441, 2012.
GONZALEZ, R. R. Infrared Radiation and Human Thermal Comfort. In: ADAIR, E. R. (Ed.).

Microwaves and Thermoregulation. New York: Academic, 1983.

HOUDAS, Y.; RING, E. F. J. Human Body Temperature, its Measurement and Regulation. New York; London: Plenum, 1982.

INSTITUTO BRASILEIRO DE GEOGRAFIA E ESTATÍSTICA. Censo Demográfico 2010: características gerais da população. Rio de Janeiro: IBGE, 2012.

INTERNATIONAL ORGANIZATION FOR STANDARDIZATION. ISO 9920: ergonomics of the thermal environment: estimation of the thermal insulation and evaporative resistance of a clothing ensemble. Genebra, 2007.

INTERNATIONAL ORGANIZATION FOR STANDARDIZATION. ISO 7726: ergonomics of the thermal environment: instruments for measuring physical quantities. Genebra, 1998.

INTERNATIONAL ORGANIZATION FOR STANDARDIZATION. ISO 10551: ergonomics of the thermal environment: assessment of the influence of the thermal environment using subjective judgment scales. Genebra, 1995.

INTERNATIONAL ORGANIZATION FOR STANDARDIZATION. ISO 8996: ergonomics of the thermal environment: determination of metabolic rate. Genebra, 2004.

JABLONSKI, N. The Evolution of Human Skin and Skin Color. Annual Review of Anthropology, v. 33, p. 585-623, 2004.

JOHANSSON, E. et al. Instruments and Methods in Outdoor Thermal Comfort Studies, the need for standardization. Urban Climate, v. 10, p. 346366, 2014.

KRÜGER, E. L. et al. Estudo de Conforto em Espaços Abertos em Região de Clima Temperado: o caso de Glasgow, Reino Unido. Ambiente Construído, Porto Alegre, v. 12, n. 1, p. 7-25, jan./mar. 2012.

KRÜGER, E. L.; DRACH, P.R.C. Impactos do uso de climatização artificial na percepção térmica em espaços abertos no centro do Rio de Janeiro. Ambiente Construído, v. 16, n. 2, p. 133-148, abr./jun. 2016.

LEE, J. Y.; TOCHIHARA, Y. Linguistic Dimensions in Descriptors Expressing Thermal Sensation in Korean: 'warm' projects thermal comfort. International Journal of

Biometeorology, v. 54, n. 4, p. 357-364, 2010. 
LEE, J. Y. et al. Issues in Combining the Categorical and Visual Analog Scale for the Assessment of Perceived Thermal Sensation: Methodological and conceptual considerations. Applied Ergonomics, v. 41, n. 2, p. 282-290, 2010.

LIN, C.; LIN, T. P.; HWANG, R. L. Thermal Comfort for Urban Parks in Subtropics: understanding visitor's perceptions, behavior and attendance. Advances in Meteorology, v. 2013.

MONTEIRO, L. M.; ALUCCI, M. P. Modelo Adaptativo de Conforto Para Avaliação in loco de Espaços Urbanos Abertos. Ambiente Construído, Porto Alegre, v. 12, n. 1, p. 61-79, jan./mar. 2012.

NARITA, C. et al. Effects of Spectral Property of Solar Radiation on Thermal Sensation at Back of Hands. In: MOVING THERMAL COMFORT STANDARDS INTO THE 21ST CENTURY, 2001. Proceedings... 2001.

NDETTO, E. L.; MATZARAKIS, A. Basic Analysis of Climate and Urban Bioclimate of Dar es Salaam, Tanzania. Theoretical and Applied Climatology, v. 114, n. 1/2, p. 213-226, 2013.

NDETTO, E. L.; MATZARAKIS, A. Urban Atmospheric Environment and Human Biometeorological Studies in Dares Salaam, Tanzania. Air Quality, Atmosphere \& Health, v. 8, n. 2, p. 175-191, 2015.
NINCE, P. C. et al. Usos dos Índices PET e UTCI na Avaliação do Conforto Termal no Campus da UFMT em Cuiabá-MT. Revista Eletrônica em Gestão, Educação e Tecnologia Ambiental, v. 9, n. 9, p. 2026-2036, 2013.

PARSONS, K. Human Thermal Environments: the effects of hot, moderate, and cold environments on human health, comfort, and performance. New York: Crc Press, 2014.

ROSSI, F. A.; KRÜGER, E. L.; BRÖDE, P. Definição de faixas de conforto e desconforto térmico para espaços abertos em Curitiba, PR, com o Índice UTCI. Ambiente Construído, Porto Alegre, v. 12, n. 1, p. 41-59, jan./mar. 2012.

TAYLOR, N. Ethnic Differences in Thermoregulation: genotypic versus phenotypic heat adaptation. Journal of Thermal Biology, v. 31, n. 1, p. 90-104, 2006.

TOCHIHARA, Y. et al. The Use of Language to Express Thermal Sensation Suggests Heat Acclimatization by Indonesian People. International Journal of Biometeorology, v. 56, n. 6, p. 1055-1064, 2012.

\section{Eduardo Leite Krüger}

Programa de Pós-Graduação em Engenharia Civil, Departamento de Construção Civil | Universidade Tecnológica Federal do Paraná | Av. Sete de Setembro, 3165, Rebouças | Curitiba - PR - Brasil | CEP 80230-901 | Tel.: (41) 3310-4725 Ramal 4725 | E-mail: ekruger@utfpr.edu.br

\section{Patricia Regina Chaves Drach}

Escola Superior de Desenho Industrial, Centro de Tecnologia e Ciências | Universidade do Estado do Rio de Janeiro | Rua Evaristo da Veiga, 95, Centro | Rio de Janeiro - RJ - Brasil | CEP 20031-040 | Tel.: (21) 2332-6908 | E-mail: patricia.drach@gmail.com

\section{Revista Ambiente Construído}

Associação Nacional de Tecnologia do Ambiente Construído

Av. Osvaldo Aranha, $99-3^{\circ}$ andar, Centro

Porto Alegre - RS - Brasil CEP $90035-190$

Telefone: +55 (51) 3308-4084

Fax: +55 (51) 3308-4054

www.seer.ufrgs.br/ambienteconstruido

E-mail: ambienteconstruido@ufrgs.br

96 Krüger, E. L.; Drach, P. R. C. 